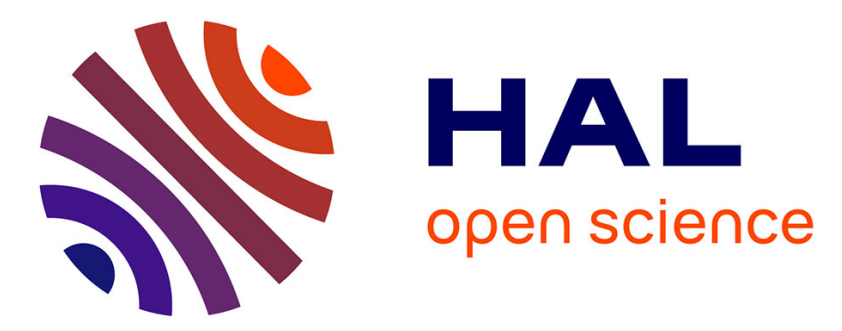

\title{
Quelques propriétés d'approximation des éléments finis de Nédélec, application à l'analyse a posteriori
}

Christine Bernardi, Frédéric Hecht

\section{To cite this version:}

Christine Bernardi, Frédéric Hecht. Quelques propriétés d'approximation des éléments finis de Nédélec, application à l'analyse a posteriori. Comptes Rendus. Mathématique, 2007, 344 (7), pp.461-466. hal00139231

\section{HAL Id: hal-00139231 \\ https://hal.science/hal-00139231}

Submitted on 30 Mar 2007

HAL is a multi-disciplinary open access archive for the deposit and dissemination of scientific research documents, whether they are published or not. The documents may come from teaching and research institutions in France or abroad, or from public or private research centers.
L'archive ouverte pluridisciplinaire HAL, est destinée au dépôt et à la diffusion de documents scientifiques de niveau recherche, publiés ou non, émanant des établissements d'enseignement et de recherche français ou étrangers, des laboratoires publics ou privés.

$$
\text { Copyright }
$$




\title{
Quelques propriétés d'approximation des éléments finis de Nédélec, application à l'analyse a posteriori
}

\author{
par Christine Bernardi et Frédéric Hecht
}

Résumé: Nous démontrons quelques propriétés d'approximation de fonctions peu régulières dans l'espace engendré par les éléments finis de Nédélec d'ordre 1, en utilisant soit l'opérateur de Nédélec usuel, soit un opérateur de type Clément adapté à ces éléments. L'application principale de ces résultats est l'analyse a posteriori de l'erreur lorsque la discrétisation fait appel à cet espace, nous en présentons un exemple.

\begin{abstract}
We prove some approximation properties of nonsmooth functions in the space constructed from Nédélec finite elements of order 1 . They rely either on the Nédélec operator or on a Clément type regularization operator linked to these elements. The main application of these results is the a posteriori analysis of the error when the discretization involves this space, we present a basic example.
\end{abstract}

Laboratoire Jacques-Louis Lions, C.N.R.S. \& Université Pierre et Marie Curie - Paris 6, B.C. 187, 4 place Jussieu, 75252 Paris Cedex 05, France.

bernardi@ann.jussieu.fr, hecht@ann.jussieu.fr 



\section{Introduction.}

Soit $\Omega$ un polyèdre de $\mathbb{R}^{3}$ que l'on suppose borné, connexe et à frontière lipschitzienne, et soit $\left(\mathcal{T}_{h}\right)_{h}$ une famille de triangulations régulière de $\Omega$, au sens usuel $[5, \S 17]$. On considère l'espace

$$
H(\operatorname{rot}, \Omega)=\left\{\boldsymbol{\varphi} \in L^{2}(\Omega)^{3} ; \operatorname{rot} \varphi \in L^{2}(\Omega)^{3}\right\} .
$$

L'espace d'éléments finis le plus simple contenu dans $H(\operatorname{rot}, \Omega)$ est construit à partir des éléments d'ordre 1 introduits par J.-C. Nédélec dans [10]:

$$
\mathbb{X}_{h}=\left\{\boldsymbol{\varphi}_{h} \in H(\operatorname{rot}, \Omega) ; \forall K \in \mathcal{T}_{h}, \varphi_{h \mid K} \in \mathcal{P}(K)\right\},
$$

où $\mathcal{P}(K)$ désigne l'espace des restrictions à $K$ des polynômes de la forme $\boldsymbol{a}+\boldsymbol{b} \times \boldsymbol{x}$, $\boldsymbol{a} \in \mathbb{R}^{3}, \boldsymbol{b} \in \mathbb{R}^{3}$. Il est donc utilisé pour la discrétisation d'un grand nombre d'équations aux dérivées partielles, intervenant par exemple en électromagnétisme ou en mécanique des fluides.

Les premières propriétés d'approximation de cet espace font appel à un opérateur d'interpolation dit de Nédélec et sont prouvées dans [10, Thm 2], mais elles demandent beaucoup trop de régularité de la fonction à approcher. Même si ces résultats ont été récemment améliorés, voir [4] et [6], nous préférons en indiquer une version optimale. Toutefois, ces résultats s'avèrent insuffisants pour un certain nombre d'applications. Nous sommes donc amenés à introduire un opérateur de régularisation de type Clément (voir [7]) adapté à l'espace $\mathbb{X}_{h}$ et à établir ses principales propriétés.

Il semble que l'analyse a posteriori de la plupart des discrétisations où apparaît l'espace $\mathbb{X}_{h}$ requiert ces nouveaux résultats. Nous présentons ici un exemple de base, concernant le calcul du potentiel vecteur, dont l'analyse a priori est effectuée dans $[1, \S 4$.a]. 


\section{Les propriétés d'approximation des éléments finis de Nédélec.}

On note $\Sigma_{h}$ l'ensemble des côtés des éléments de $\mathcal{T}_{h}$. L'opérateur de Nédélec à valeurs dans $\mathbb{X}_{h}$ est défini de façon unique par les relations

$$
\forall \sigma \in \Sigma_{h}, \quad \int_{\sigma}\left(\mathcal{N}_{h} \varphi\right)(\boldsymbol{\tau}) \cdot \boldsymbol{t} d \boldsymbol{\tau}=\int_{\sigma} \boldsymbol{\varphi}(\boldsymbol{\tau}) \cdot \boldsymbol{t} d \boldsymbol{\tau}
$$

où $\boldsymbol{t}$ est un vecteur tangent à $\sigma$, voir [10, Thm 1]. Nous ne donnons pas la démonstration du résultat suivant, car

(i) la première estimation se démontre par les mêmes arguments que dans [6, Lemma 3.3], plus précisément grâce à la construction d'un opérateur $\mathcal{R}_{h}$ tel que $\operatorname{rot}\left(\mathcal{N}_{h} \varphi\right)=$ $\mathcal{R}_{h}(\operatorname{rot} \varphi)$,

(ii) la second estimation utilise le fait que l'opérateur $\mathcal{N}_{h}$ préserve les constantes sur chaque élément $K$ de $\mathcal{T}_{h}$ et la propriété de continuité de cet opérateur établie dans [1, Lemma 4.7].

Proposition 1. On a les estimations suivantes

(i) pour toute fonction $\varphi$ de $H(\operatorname{rot}, \Omega)$ telle que $\operatorname{rot} \varphi$ appartienne à $H^{s}(\Omega)^{3}, 0<s \leq 1$,

$$
\left\|\operatorname{rot}\left(\boldsymbol{\varphi}-\mathcal{N}_{h} \boldsymbol{\varphi}\right)\right\|_{L^{2}(\Omega)^{3}} \leq c h^{s}\|\operatorname{rot} \varphi\|_{H^{s}(\Omega)^{3}},
$$

(ii) pour toute fonction $\varphi$ de $H(\operatorname{rot}, \Omega) \cap H^{s}(\Omega)^{3}, \frac{1}{2}<s \leq 1$, telle que $\operatorname{rot} \varphi$ appartienne à $H^{t}(\Omega)^{3}, t>0$,

$$
\left\|\varphi-\mathcal{N}_{h} \varphi\right\|_{L^{2}(\Omega)^{3}} \leq c h^{s}\left(\|\varphi\|_{H^{s}(\Omega)^{3}}+\|\operatorname{rot} \varphi\|_{H^{t}(\Omega)^{3}}\right) .
$$

Toujours d'après [10, Thm 1], il existe une base de $\mathbb{X}_{h}$ formée par les fonctions $\boldsymbol{\xi}_{\sigma}$, $\sigma \in \Sigma_{h}$, telles que

$$
\int_{\sigma} \boldsymbol{\xi}_{\sigma}(\boldsymbol{\tau}) \cdot \boldsymbol{t} d \boldsymbol{\tau}=1 \quad \text { et } \quad \forall \sigma^{\prime} \in \Sigma_{h}, \sigma^{\prime} \neq \sigma, \quad \int_{\sigma^{\prime}} \boldsymbol{\xi}_{\sigma}(\boldsymbol{\tau}) \cdot \boldsymbol{t} d \boldsymbol{\tau}=0 .
$$

On peut noter que l'opérateur $\mathcal{N}_{h}$ défini par les relations (3) vérifie

$$
\mathcal{N}_{h} \varphi=\sum_{\sigma \in \Sigma_{h}}\left(\int_{\sigma} \varphi(\boldsymbol{\tau}) \cdot \boldsymbol{t} d \boldsymbol{\tau}\right) \boldsymbol{\xi}_{\sigma}
$$

À tout élément $\sigma$ de $\Sigma_{h}$, on associe maintenant un tétraèdre $K_{\sigma}$ de $\mathcal{T}_{h}$ qui contient $\sigma$ et on désigne par $\pi_{\sigma}$ l'opérateur de projection orthogonale de $L^{2}\left(K_{\sigma}\right)^{3}$ sur $\mathcal{P}_{0}\left(K_{\sigma}\right)^{3}$, où $\mathcal{P}_{0}(K)$ est l'espace des fonctions constantes sur $K$. On introduit l'opérateur modifié $\mathcal{N}_{h}^{*}$ défini par

$$
\mathcal{N}_{h}^{*} \boldsymbol{\varphi}=\sum_{\sigma \in \Sigma_{h}}\left(\int_{\sigma}\left(\pi_{\sigma} \boldsymbol{\varphi}\right)(\boldsymbol{\tau}) \cdot \boldsymbol{t} d \boldsymbol{\tau}\right) \boldsymbol{\xi}_{\sigma}
$$

Pour tout élément $K$ de $\mathcal{T}_{h}$ et pour toute face $e$ de $K, h_{K}$ et $h_{e}$ désignent les diamètres de $K$ et de $e$. D'autre part, on note $\Delta_{K}$, resp. $\Delta_{e}$, l'union des éléments de $\mathcal{T}_{h}$ qui partagent au moins un côté avec $K$, resp. avec $e$. 
Proposition 2. On a les estimations suivantes

(i) pour toute fonction $\varphi$ de $H(\operatorname{rot}, \Omega) \cap H^{s}(\Omega)^{3}, 0 \leq s \leq 1$, et pour tout élément $K$ de $\mathcal{T}_{h}$,

$$
\left\|\boldsymbol{\varphi}-\mathcal{N}_{h}^{*} \boldsymbol{\varphi}\right\|_{L^{2}(K)^{3}} \leq c h_{K}^{s}\|\boldsymbol{\varphi}\|_{H^{s}\left(\Delta_{K}\right)^{3}},
$$

(ii) pour toute fonction $\varphi$ de $H(\operatorname{rot}, \Omega) \cap H^{s}(\Omega)^{3}, \frac{1}{2}<s \leq 1$, pour tout élément $K$ de $\mathcal{T}_{h}$ et pour toute face $e$ de $K$,

$$
\left\|\boldsymbol{\varphi}-\mathcal{N}_{h}^{*} \boldsymbol{\varphi}\right\|_{L^{2}(e)^{3}} \leq c h_{e}^{s-\frac{1}{2}}\|\boldsymbol{\varphi}\|_{H^{s}\left(\Delta_{e}\right)^{3}}
$$

Démonstration: Nous prouvons la majoration (8) tout d'abord pour $s=0$, puis pour $s=1$, le résultat général s'en déduisant par un argument d'interpolation standard. Puis nous démontrons l'estimation (9).

1) Si $F_{K}$ désigne une application affine qui envoie le tétraèdre de référence $\hat{K}$ sur $K$ et $B_{K}$ est sa matrice jacobienne, on pose: $\hat{\boldsymbol{\xi}}_{\sigma}=B_{K}^{T} \boldsymbol{\xi}_{\sigma} \circ F_{K}$. On note $\widehat{\Sigma}$ l'ensemble des côtés de $\hat{K}$ et $\hat{\sigma}=F_{K}^{-1}(\sigma)$. On vérifie alors facilement que

$$
\int_{\hat{\sigma}} \hat{\boldsymbol{\xi}}_{\sigma}(\hat{\boldsymbol{\tau}}) \cdot \hat{\boldsymbol{t}} d \hat{\boldsymbol{\tau}}=1 \quad \text { and } \quad \forall \hat{\sigma}^{\prime} \in \widehat{\Sigma}, \hat{\sigma}^{\prime} \neq \hat{\sigma}, \quad \int_{\hat{\sigma}^{\prime}} \hat{\boldsymbol{\xi}}_{\sigma}(\hat{\boldsymbol{\tau}}) \cdot \hat{\boldsymbol{t}} d \hat{\boldsymbol{\tau}}=0
$$

Par conséquent, les $\hat{\boldsymbol{\xi}}_{\sigma}$ coïncident avec les fonctions de base $\boldsymbol{\xi}_{\hat{\sigma}}$ de l'élément fini sur $\hat{K}$ et leurs normes sont donc bornées indépendamment de $K$. En passant au domaine de référence, on obtient ainsi

$$
\left\|\boldsymbol{\xi}_{\sigma}\right\|_{L^{2}(K)} \leq c h_{K}^{\frac{1}{2}}, \quad\left\|\boldsymbol{\xi}_{\sigma}\right\|_{L^{2}(e)} \leq c .
$$

D'autre part, on voit grâce à une inégalité de Cauchy-Schwarz que

$$
\left|\int_{\sigma}\left(\pi_{\sigma} \boldsymbol{\varphi}\right)(\boldsymbol{\tau}) \cdot \boldsymbol{t} d \boldsymbol{\tau}\right| \leq c h_{K}^{\frac{1}{2}}\left\|\pi_{\sigma} \boldsymbol{\varphi}\right\|_{L^{2}(\sigma)^{3}}
$$

On pose $\hat{\boldsymbol{\varphi}}=\boldsymbol{\varphi} \circ F_{K_{\sigma}}$ et on note $\hat{\pi}$ l'opérateur de projection orthogonale de $L^{2}(\hat{K})^{3}$ sur $\mathcal{P}_{0}(\hat{K})^{3}$. On déduit de ce qui précède que

$$
\left|\int_{\sigma}\left(\pi_{\sigma} \boldsymbol{\varphi}\right)(\boldsymbol{\tau}) \cdot \boldsymbol{t} d \boldsymbol{\tau}\right| \leq c h_{K}\|\hat{\pi} \hat{\boldsymbol{\varphi}}\|_{L^{2}(\hat{\sigma})^{3}}
$$

L'équivalence des normes sur $\mathcal{P}_{0}(\hat{K})^{3}$ entraîne que

$$
\left|\int_{\sigma}\left(\pi_{e} \boldsymbol{\varphi}\right)(\boldsymbol{\tau}) \cdot \boldsymbol{t} d \boldsymbol{\tau}\right| \leq c h_{K}\|\hat{\pi} \hat{\boldsymbol{\varphi}}\|_{L^{2}(\hat{K})^{3}} \leq c h_{K}\|\hat{\boldsymbol{\varphi}}\|_{L^{2}(\hat{K})^{3}} \leq c^{\prime} h_{K_{\sigma}^{-\frac{1}{2}}}^{-\boldsymbol{\varphi} \|_{L^{2}\left(K_{\sigma}\right)^{3}}}
$$

En combinant ceci avec (10) et en utilisant la régularité de la famille de triangulations, on en déduit

$$
\left\|\mathcal{N}_{h}^{*} \boldsymbol{\varphi}\right\|_{L^{2}(K)^{3}} \leq c\|\boldsymbol{\varphi}\|_{L^{2}\left(\Delta_{K}\right)^{3}} .
$$


Grâce à une inégalité triangulaire, ceci donne (8) pour $s=0$.

2) Soit $\bar{\varphi}$ une fonction constante sur $\Delta_{K}$. Comme $\left(\mathcal{N}_{h}^{*} \bar{\varphi}\right)_{\mid K}$ est égal à $\bar{\varphi}_{\mid K}$, on a

$$
\left\|\boldsymbol{\varphi}-\mathcal{N}_{h}^{*} \boldsymbol{\varphi}\right\|_{L^{2}(K)^{3}} \leq\|\boldsymbol{\varphi}-\overline{\boldsymbol{\varphi}}\|_{L^{2}(K)^{3}}+\left\|\mathcal{N}_{h}^{*}(\boldsymbol{\varphi}-\overline{\boldsymbol{\varphi}})\right\|_{L^{2}(K)^{3}} .
$$

On déduit alors de (11) que

$$
\left\|\boldsymbol{\varphi}-\mathcal{N}_{h}^{*} \boldsymbol{\varphi}\right\|_{L^{2}(K)^{3}} \leq c\|\boldsymbol{\varphi}-\overline{\boldsymbol{\varphi}}\|_{L^{2}\left(\Delta_{K}\right)^{3}} .
$$

La majoration (8) pour $s=1$ est alors une conséquence de [9, Thm 7.1] (voir aussi [2, Lemma IX.3.6]).

3) Finalement les mêmes arguments que dans la partie 1) de la démonstration et la seconde estimation dans (10) entraînent

$$
\left\|\mathcal{N}_{h}^{*} \varphi\right\|_{L^{2}(e)^{3}} \leq c h_{e}^{-\frac{1}{2}}\|\varphi\|_{L^{2}\left(\Delta_{e}\right)^{3}} .
$$

Là encore, en utilisant l'égalité $\left(\mathcal{N}_{h}^{*} \bar{\varphi}\right)_{\mid e}=\bar{\varphi}_{\mid e}$ pour toute fonction constante sur $\Delta_{e}$, on déduit de (12) que

$$
\left\|\boldsymbol{\varphi}-\mathcal{N}_{h}^{*} \boldsymbol{\varphi}\right\|_{L^{2}(e)^{3}} \leq\|\boldsymbol{\varphi}-\overline{\boldsymbol{\varphi}}\|_{L^{2}(e)^{3}}+c h_{e}^{-\frac{1}{2}}\|\boldsymbol{\varphi}-\bar{\varphi}\|_{L^{2}\left(\Delta_{e}\right)^{3}} .
$$

Si $e$ est inclus dans deux éléments $K$ et $K^{\prime}$ de $\mathcal{T}_{h}$, on vérifie facilement en prenant $\bar{\varphi}$ égal à la valeur moyenne de $\varphi$ sur $e$ et en passant à l'élément de référence que, pour $s>\frac{1}{2}$,

$$
\|\boldsymbol{\varphi}-\overline{\boldsymbol{\varphi}}\|_{L^{2}(e)^{3}}+h_{K}^{-\frac{1}{2}}\|\boldsymbol{\varphi}-\overline{\boldsymbol{\varphi}}\|_{L^{2}\left(K \cup K^{\prime}\right)^{3}} \leq c h_{K}^{s-\frac{1}{2}}\|\boldsymbol{\varphi}\|_{H^{s}\left(K \cup K^{\prime}\right)} .
$$

De plus, en utilisant une fois de plus [9, Thm 7.1], on peut étendre ce résultat de $K \cup K^{\prime}$ à $\Delta_{e}$. Ceci donne la majoration (9).

Remarque. Considérons les espaces

$$
H_{0}(\operatorname{rot}, \Omega)=\{\boldsymbol{\varphi} \in H(\operatorname{rot}, \Omega) ; \boldsymbol{\varphi} \times \boldsymbol{n}=0 \text { sur } \partial \Omega\}, \quad \mathbb{X}_{h}^{0}=\mathbb{X}_{h} \cap H_{0}(\operatorname{rot}, \Omega) .
$$

L'opérateur $\mathcal{N}_{h}$ envoie $H_{0}(\operatorname{rot}, \Omega)$ dans $\mathbb{X}_{h}^{0}$, mais ce n'est plus le cas pour $\mathcal{N}_{h}^{*}$. On peut toutefois construire par les moyens habituels (voir $[2, \S I X .3]$ ) un opérateur $\mathcal{N}_{h}^{0}$ à valeurs dans $\mathbb{X}_{h}^{0}$ vérifiant les mêmes propriétés (8) et $(9)$ que $\mathcal{N}_{h}^{*}$, mais seulement pour les fonctions de $H_{0}(\operatorname{rot}, \Omega)$. 


\section{Un exemple d'application à l'analyse a posteriori.}

On suppose pour simplifier que $\Omega$ est simplement connexe et a une frontière connexe. Dans ce cas, un champ de vecteurs $\boldsymbol{u}$ dans $L^{2}(\Omega)^{3}$ vérifie

$$
\operatorname{div} \boldsymbol{u}=0 \quad \operatorname{dans} \Omega, \quad \boldsymbol{u} \cdot \boldsymbol{n}=0 \quad \text { sur } \partial \Omega,
$$

si et seulement s'il existe un vecteur potentiel $\boldsymbol{\psi}$ tel que

$$
\boldsymbol{u}=\operatorname{rot} \boldsymbol{\psi} \quad \operatorname{dans} \Omega, \quad \operatorname{div} \boldsymbol{\psi}=0 \quad \operatorname{dans} \Omega, \quad \boldsymbol{\psi} \times \boldsymbol{n}=0 \quad \text { sur } \partial \Omega,
$$

voir [1, Thm 3.17]. La fonction $\boldsymbol{\psi}$ est alors partie de la solution du problème variationnel suivant

Trouver $(\boldsymbol{\psi}, \theta)$ in $H_{0}(\boldsymbol{r o t}, \Omega) \times H_{0}^{1}(\Omega)$ tel que

$$
\begin{gathered}
\forall \boldsymbol{\varphi} \in H_{0}(\operatorname{rot}, \Omega), \quad \int_{\Omega} \operatorname{rot} \psi \cdot \operatorname{rot} \varphi d x+\int_{\Omega} \boldsymbol{\varphi} \cdot \operatorname{grad} \theta d \boldsymbol{x}=\int_{\Omega} \boldsymbol{u} \cdot \operatorname{rot} \boldsymbol{\varphi} d \boldsymbol{x}, \\
\forall \mu \in H_{0}^{1}(\Omega), \quad \int_{\Omega} \psi \cdot \operatorname{grad} \mu d \boldsymbol{x}=0 .
\end{gathered}
$$

Il est prouvé dans $[1, \S 4 . a]$ que la première forme bilinéaire est elliptique sur le noyau

$$
\left\{\varphi \in H_{0}(\operatorname{rot}, \Omega) ; \forall \mu \in H_{0}^{1}(\Omega), \int_{\Omega} \boldsymbol{\varphi} \cdot \operatorname{grad} \mu d \boldsymbol{x}=0\right\},
$$

et que la seconde vérifie une condition inf-sup, de sorte que ce problème admet une solution unique. De plus, on constate que l'inconnue $\theta$ est nulle.

Soit $\mathbb{M}_{h}$ l'espace des fonctions de $H_{0}^{1}(\Omega)$ dont la restriction à chaque élément $K$ de $\mathcal{T}_{h}$ est affine. On considère le problème discret suivant

$$
\begin{aligned}
& \text { Trouver }\left(\boldsymbol{\psi}_{h}, \theta_{h}\right) \text { in } \mathbb{X}_{h}^{0} \times \mathbb{M}_{h} \text { tel que } \\
& \forall \boldsymbol{\varphi}_{h} \in \mathbb{X}_{h}^{0}, \quad \int_{\Omega} \operatorname{rot} \boldsymbol{\psi}_{h} \cdot \operatorname{rot} \boldsymbol{\varphi}_{h} d \boldsymbol{x}+\int_{\Omega} \boldsymbol{\varphi}_{h} \cdot \operatorname{grad} \theta_{h} d \boldsymbol{x}=\int_{\Omega} \boldsymbol{u} \cdot \operatorname{rot} \boldsymbol{\varphi}_{h} d \boldsymbol{x}, \\
& \forall \mu_{h} \in \mathbb{M}_{h}, \quad \int_{\Omega} \boldsymbol{\psi}_{h} \cdot \operatorname{grad} \mu_{h} d \boldsymbol{x}=0 .
\end{aligned}
$$

Là encore, il est prouvé dans [1, Prop. 4.11] que ce problème admet une solution unique $\left(\boldsymbol{\psi}_{h}, \theta_{h}\right)$, avec $\theta_{h}=0$. Une estimation d'erreur a priori entre les solutions des problèmes (16) et (17) est établie dans [1, Thm 4.13]. $\mathbb{X}_{h}^{0}$

On écrit tout d'abord l'équation du résidu, pour tous $\varphi$ dans $H_{0}(\operatorname{rot}, \Omega)$ et $\varphi_{h}$ dans

$$
\begin{aligned}
\int_{\Omega} \operatorname{rot}\left(\psi-\psi_{h}\right) \cdot \operatorname{rot} \varphi d x & +\int_{\Omega} \varphi \cdot \operatorname{grad}\left(\theta-\theta_{h}\right) d x \\
& =\int_{\Omega}\left(u-\operatorname{rot} \psi_{h}\right) \cdot \operatorname{rot}\left(\varphi-\varphi_{h}\right) d x
\end{aligned}
$$


En outre, on remarque que:

(i) si $\mu$ désigne la solution dans $H_{0}^{1}(\Omega)$ du problème

$$
\forall \chi \in H_{0}^{1}(\Omega), \quad \int_{\Omega} \operatorname{grad} \mu \cdot \operatorname{grad} \chi d x=\int_{\Omega} \varphi \cdot \operatorname{grad} \chi d x,
$$

la fonction $\tilde{\varphi}=\boldsymbol{\varphi}-\operatorname{grad} \mu$ appartient à $H_{0}(\operatorname{rot}, \Omega)$ et est à divergence nulle;

(ii) on déduit de $[8, \S 2 . \mathrm{B}]$ (voir aussi [3]) qu'il existe un fonction $\varphi^{*}$ de $H^{1}(\Omega)^{3}$ telle que $\tilde{\varphi}-\varphi^{*}$ soit un gradient et qui vérifie

$$
\left\|\varphi^{*}\right\|_{H^{1}(\Omega)^{3}} \leq c\|\tilde{\varphi}\|_{H(\operatorname{rot}, \Omega)} \leq c^{\prime}\|\boldsymbol{\varphi}\|_{H(\operatorname{rot}, \Omega)} .
$$

On peut donc rempacer $\varphi$ par $\varphi^{*}$ dans (18). La seconde équation du résidu s'écrit, pour tous $\mu$ dans $H_{0}^{1}(\Omega)$ et $\mu_{h}$ dans $\mathbb{M}_{h}$,

$$
\int_{\Omega}\left(\psi-\psi_{h}\right) \cdot \operatorname{grad} \mu d x=-\int_{\Omega} \psi_{h} \cdot \operatorname{grad}\left(\mu-\mu_{h}\right) d x .
$$

Par intégration par parties, en prenant $\varphi_{h}$ égal à $\mathcal{N}_{h}^{0} \varphi^{*}$ pour l'opérateur $\mathcal{N}_{h}^{0}$ introduit en remarque et $\mu_{h}$ égal à l'image de $\mu$ par un opérateur de régularisation standard (voir [2, Thm IX.3.11] par exemple), on déduit de (18) et (19) le résultat suivant. Étant donnée une approximation $\boldsymbol{u}_{h}$ de $\boldsymbol{u}$ dans $\mathbb{X}_{h}$, on définit pour chaque élément $K$ de $\mathcal{T}_{h}$ l'indicateur d'erreur

$$
\begin{aligned}
\eta_{K}=h_{K}\left\|\operatorname{rot} \boldsymbol{u}_{h}\right\|_{L^{2}(K)^{3}}+\sum_{e \in \mathcal{E}_{K}} h_{e}^{\frac{1}{2}}\left\|\left[\operatorname{rot} \boldsymbol{\psi}_{h} \times \boldsymbol{n}\right]_{e}\right\|_{L^{2}(e)^{3}} \\
+h_{K}\left\|\operatorname{div} \boldsymbol{\psi}_{h}\right\|_{L^{2}(K)}+\sum_{e \in \mathcal{E}_{K}} h_{e}^{\frac{1}{2}}\left\|\left[\boldsymbol{\psi}_{h} \cdot \boldsymbol{n}\right]_{e}\right\|_{L^{2}(e)}
\end{aligned}
$$

où $\mathcal{E}_{K}$ désigne l'ensemble des faces de $K$ qui ne sont pas contenues dans $\partial \Omega$ et $[\cdot]_{e}$ le saut à travers un élément $e$ de $\mathcal{E}_{K}$. En vue de simplifier la mise en ouvre, on peut bien sûr remplacer $\left[\boldsymbol{r o t} \boldsymbol{\psi}_{h} \times \boldsymbol{n}\right]_{e}$ par $\left[\boldsymbol{\operatorname { r o t }} \boldsymbol{\psi}_{h}\right]_{e}$ et $\left[\boldsymbol{\psi}_{h} \cdot \boldsymbol{n}\right]_{e}$ par $\left[\boldsymbol{\psi}_{h}\right]_{e}$ dans la définition de $\eta_{K}$.

Proposition 3. Pour toute fonction $\boldsymbol{u}$ de $H(\operatorname{rot}, \Omega)$, on a l'estimation a posteriori suivante entre les solutions $(\boldsymbol{\psi}, \theta)$ du problème (16) et $\left(\boldsymbol{\psi}_{h}, \theta_{h}\right)$ du problème (17)

$$
\left\|\boldsymbol{\psi}-\boldsymbol{\psi}_{h}\right\|_{H(\operatorname{rot}, \Omega)} \leq c\left(\sum_{K \in \mathcal{T}_{h}}\left(\eta_{K}^{2}+h_{K}^{2}\left\|\operatorname{rot}\left(\boldsymbol{u}-\boldsymbol{u}_{h}\right)\right\|_{L^{2}(K)^{3}}^{2}\right)\right)^{\frac{1}{2}} .
$$

On peut également, par des choix appropriés de $\varphi$ dans (18) et $\mu$ dans (19) majorer chaque indicateur $\eta_{K}$ par une constante fois l'erreur dans un voisinage de $K$. L'estimation que nous avons prouvée est donc parfaitement optimale et les $\eta_{K}, K \in \mathcal{T}_{h}$, constituent un outil efficace pour la construction d'un maillage adapté à la solution $\psi$. 


\section{Références bibliographiques}

[1] C. Amrouche, C. Bernardi, M. Dauge, V. Girault - Vector potentials in threedimensional nonsmooth domains, Math. Meth. in the Applied Sciences 21 (1998), 823-864.

[2] C. Bernardi, Y. Maday, F. Rapetti - Discrétisations variationnelles de problèmes aux limites elliptiques, Collection "Mathématiques et Applications" 45, Springer-Verlag (2004).

[3] M.Sh. Birman, M.Z. Solomyak - $L^{2}$-theory of the Maxwell operator in arbitrary domains, Russ. Math. Surv. 42 (1987), 75-96.

[4] A. Buffa, M. Costabel, M. Dauge - Algebraic convergence for anisotropic edge elements in polyhedral domains, Numer. Math. 101 (2005), 29-65.

[5] P.G. Ciarlet - Basic Error Estimates for Elliptic Problems, Handbook of Numerical Analysis, Vol. II, édité par P.G. Ciarlet \& J.-L. Lions (1989).

[6] P. Ciarlet Jr., J. Zou — Fully discrete finite element approaches for time-dependent Maxwell's equations, Numer. Math. 82 (1999), 193-219.

[7] P. Clément - Approximation by finite element functions using local regularization, R.A.I.R.O. Anal. Numér. 9 R2 (1975), 77-84.

[8] M. Costabel, M. Dauge - Computation of resonance frequencies for Maxwell equations in non smooth domains, in Topics in Computational Wave Propagation, M. Ainsworth, P. Davies, D. Duncan, P. Martin and B. Rynne eds., Springer (2004), $125-161$.

[9] T. Dupont, R. Scott - Polynomial approximation of functions in Sobolev spaces, Math. Comput. 34 (1980), 441-463.

[10] J.-C. Nédélec — Mixed finite elements in $\mathbb{R}^{3}$, Numer. Math. 35 (1980), 315-341. 


\section{Abridged English Version \\ Some further approximation properties of Nédélec finite elements, application to a posteriori analysis}

\section{Introduction}

Let $\Omega$ be a bounded connected polyhedron in $\mathbb{R}^{3}$, and let $\left(\mathcal{T}_{h}\right)_{h}$ be a regular family of triangulations of $\Omega$, in the sense of $[5, \S 17]$. The curl operator being here denoted by rot, its domain $H($ rot, $\Omega)$ is defined in (1). The simplest finite element space contained in $H($ rot, $\Omega)$, which relies on the finite elements introduced by J.-C. Nédélec in [10], is the space $\mathbb{X}_{h}$ defined in (2), where $\mathcal{P}(K)$ stands for the space of restrictions to $K$ of polynomials of the form $\boldsymbol{a}+\boldsymbol{b} \times \boldsymbol{x}, \boldsymbol{a} \in \mathbb{R}^{3}, \boldsymbol{b} \in \mathbb{R}^{3}$. This space is used for the discretization of a large number of partial differential equations, issued from electromagnetism or fluid mechanics for instance.

The first approximation properties involve the Nédélec interpolation operator and are established in [10, Thm 2], but they require too much regularity on the function to be approximated. Even if these results have been improved, see [4] and [6], we have rather state an optimal version. However, this does not seem sufficient for a number of applications, so we introduce a Clément [7] type regularization operator which is appropriate for the space $\mathbb{X}_{h}$ and prove its main properties. The a posteriori analysis of most discretizations which involve the space $\mathbb{X}_{h}$ require these new results. We present a basic example concerning the vector potential problem, see [1, §4.a].

\section{The approximation properties of Nédélec finite elements}

Let $\Sigma_{h}$ denote the set of edges of all tetrahedra in $\mathcal{T}_{h}$. It follows from [10, Thm 1] that the conditions in (3) define an operator $\mathcal{N}_{h}$ with values in $\mathbb{X}_{h}$ in a unique way. We refer to [6, Lemma 3.3] for the main arguments of the proof of the next results.

Proposition 1. Estimate (4) holds for any function $\varphi$ in $H(\operatorname{rot}, \Omega)$ such that $\operatorname{rot} \varphi$ belongs to $H^{s}(\Omega)^{d}, 0<s \leq 1$. Estimate (5) holds for any function $\varphi$ in $H(\operatorname{rot}, \Omega) \cap H^{s}(\Omega)^{3}$, $\frac{1}{2}<s \leq 1$, such that $\operatorname{rot} \varphi$ belongs to $H^{t}(\Omega)^{3}, t>0$.

For the functions $\boldsymbol{\xi}_{\sigma}$ introduced in (6), we consider the modified operator $\mathcal{N}_{h}^{*}$ defined in (7), where, for each $\sigma$ in $\Sigma_{h}, K_{\sigma}$ denotes a tetrahedron in $\mathcal{T}_{h}$ containing $\sigma$ and $\pi_{\sigma}$ stands for the orthogonal projection operator from $L^{2}\left(K_{\sigma}\right)^{3}$ onto the space of constant vector fields on $K_{\sigma}$. The next statement requires some notation: for each $K$ in $\mathcal{T}_{h}$ and each face $e$ of $K, h_{K}$ and $h_{e}$ are the diameters of $K$ and $e ; \Delta_{K}$ and $\Delta_{e}$ are the union of elements of $\mathcal{T}_{h}$ that share at least an edge with $K$ and $e$, respectively. We refer to [2, $\S I X .3]$ for the main ideas of its proof.

Proposition 2. Estimate (8) holds for any function $H(\operatorname{rot}, \Omega) \cap H^{s}(\Omega)^{3}, 0 \leq s \leq 1$, and for any element $K$ of $\mathcal{T}_{h}$. Estimate (9) holds for any function $\varphi$ in $H($ rot, $\Omega) \cap H^{s}(\Omega)^{3}$, $\frac{1}{2}<s \leq 1$, for any element $K$ of $\mathcal{T}_{h}$ and any face $e$ of $K$. 
The spaces $H_{0}(\operatorname{rot}, \Omega)$ and $\mathbb{X}_{h}^{0}$ being defined in (13), it can be noted that, in contrast with $\mathcal{N}_{h}$, the operator $\mathcal{N}_{h}^{*}$ does not map $H_{0}(\operatorname{rot}, \Omega)$ into $\mathbb{X}_{h}^{0}$. However, an operator $\mathcal{N}_{h}^{0}$ which satisfies this property can easily be constructed and still satisfies estimates (8) and (9), now for functions $\varphi$ in $H_{0}(\operatorname{rot}, \Omega)$.

\section{An example of application to a posteriori analysis}

From now on, we assume for simplicity that $\Omega$ is simply-connected and has a connected boundary. Thus, we recall from [1, Thm 3.17] that a vector field $\boldsymbol{u}$ in $L^{2}(\Omega)^{3}$ satisfies (14) if and only if there exists a vector potential $\boldsymbol{\psi}$ such that (15) holds. Equation (15) admits the equivalent variational formulation (16), and this problem has a unique solution $(\boldsymbol{\psi}, \theta)$, with $\theta=0$.

Let $\mathbb{M}_{h}$ denote the space of functions in $H_{0}^{1}(\Omega)$ such that their restrictions to each element of $\mathcal{T}_{h}$ are affine. We consider the discrete problem (17) and recall from [1, Prop. 4.11] that it is well-posed and that the part $\theta_{h}$ of its solution is zero. We also refer to [1, Thm 4.13] for an a priori estimate of the error issued from this discretization.

The first residual equation (18) is valid for all $\boldsymbol{\varphi}$ in $H_{0}(\operatorname{rot}, \Omega)$ and $\boldsymbol{\varphi}_{h}$ in $\mathbb{X}_{h}^{0}$, the second equation (19) is valid for all $\mu$ in $H_{0}^{1}(\Omega)$ and $\mu_{h}$ in $\mathbb{M}_{h}$. Combining them and using the previously introduced operator $\mathcal{N}_{h}^{0}$ and also a standard regularization operator with values in $\mathbb{M}_{h}[2$, Thm IX.3.11] leads to the final result. We introduce an approximation $\boldsymbol{u}_{h}$ of $\boldsymbol{u}$ in $\mathbb{X}_{h}$ and define the error indicators $\eta_{K}, K \in \mathcal{T}_{h}$, by (20), where $\mathcal{E}_{K}$ denotes the set of faces of $K$ which are not contained in $\partial \Omega$ and $[\cdot]_{e}$ the jump through $e$.

Proposition 3. For any vector field $\boldsymbol{u}$ in $H($ rot, $\Omega)$, the a posteriori error estimate $(21)$ holds between the solutions $(\boldsymbol{\psi}, \theta)$ of problem $(16)$ and $\left(\boldsymbol{\psi}_{h}, \theta_{h}\right)$ of problem (17). 